Research Article

\title{
Prediction of Mechanical Strength Based on Deep Learning Using the Scanning Electron Image of Microscopic Cemented Paste Backfill
}

\author{
Xuebin Qin $\mathbb{D}^{1},{ }^{1}$ Shifu Cui $\mathbb{D}^{2},{ }^{2}$ Lang Liu $\mathbb{D}^{1},{ }^{1}$ Pai Wang, ${ }^{1}$ Mei Wang ${ }^{\mathbb{D}},{ }^{1}$ and Jie Xin ${ }^{1}$ \\ ${ }^{1}$ Xi'an University of Science and Technology, 58 Yanta Rd., Xi'an, Shaanxi, China \\ ${ }^{2}$ Ankang Highway Bureau of Shaanxi Province, Ankang 725000, Shaanxi, China \\ Correspondence should be addressed to Shifu Cui; 13689101111@163.com
}

Received 22 February 2018; Accepted 14 June 2018; Published 1 November 2018

Academic Editor: Chiara Bedon

Copyright (c) 2018 Xuebin Qin et al. This is an open access article distributed under the Creative Commons Attribution License, which permits unrestricted use, distribution, and reproduction in any medium, provided the original work is properly cited.

The mechanical strength of cemented backfill is an important indicator in mining filling. To study the nonlinear relationship between cemented paste backfill (CPB) and mechanical response, a deep learning technique is employed to establish the end-toend mapping relationship between the scanning electron microscope (SEM) images and mechanical strength. A seven-layer convolution neural network is set up in the experiment, and the relationship between the SEM image and mechanical strength is established. In addition, the difference between the measured and predicted values is calculated and the mean and variance of the error are analyzed. The average accuracy of the mechanical strength prediction is found to be $8.28 \%$. Thus, the proposed method provides a new technique for the quantitative analysis of mechanical strength of microscale CPB.

\section{Introduction}

With the development of mineral resources, the requirements for energy conservation, emission reduction, and environmental protection have become increasingly stringent. Hence, achieving nonwaste clean mining is inevitable for the future growth of mining. To realize safe production, filling technology has become the most suitable choice for deep mining and is its foreseeable direction of development. It is also an important technical route for achieving safe and clean mining with a high efficiency. Filling technology offers a reliable technical support for the coordinated development of safe production and environment in the mining industry, which not only creates conditions for the expansion and efficiency of mines but also reduces the pressure of the tailings discharge on the surface. It is conducive to environmental protection and promotes energy conservation and emission reduction in mines. It facilitates the synergetic growth for the economic, social, and eco-environmental benefits of mining. The mechanical strength of the cemented paste backfill (CPB) is an important aspect of filling technology. If the strength of the filling body is extremely high, it will cause waste. If the strength of the CPB is insufficient, it may cause a safety accident. Various national and foreign scholars have also conducted numerous research studies related to these problems. Professor Fei studied the energy change and damage mechanism of uniaxial compression of cemented tailings backfill based on the characteristics of infrasonic signals [1]. A neural network was employed to obtain the optimized proportion of cemented tailings by Qinli et al. of Central South University. In another study, by considering the concentration of the slurry and amount of each component as input factors while regarding the slump, $7 \mathrm{~d}$ (day) compressive strength, and $28 \mathrm{~d}$ compressive strength as output factors, a back propagation (BP) neural network was used to establish the prediction model for training and testing the samples [2]. Xu et al. studied the electrical resistivity of $\mathrm{CPB}$ and performed a uniaxial compressive strength test for $\mathrm{CPB}$ during consolidation under varying conditions of the ash-sand mass ratio, slurry mass fraction, and curing age. $\mathrm{Xu}$ et al. found that the resistivity characteristics and strength of the correlation revealed the mechanism of the resistivity variation throughout the consolidation of the cemented backfill [3]. Zhou et al. of Zhejiang University analyzed the variation in the soil pore 
TABle 1: Test of specific gravity, loose bulk density, dense bulk density, porosity, and natural rest angle.

\begin{tabular}{lccccc}
\hline Material & Specific gravity & Loose bulk density $\left(\mathrm{t} / \mathrm{m}^{3}\right)$ & Dense bulk density $\left(\mathrm{t} / \mathrm{m}^{3}\right)$ & Porosity $(\%)$ & Natural rest angle $\left({ }^{\circ}\right)$ \\
\hline Full tailings & 2.992 & 1.392 & 1.955 & 34.659 & 42.997 \\
\hline
\end{tabular}

TABLE 2: Full-tail grading composition.

\begin{tabular}{lcccccrrr}
\hline Chemical composition & $\mathrm{TFe}$ & $\mathrm{SiO}_{2}$ & $\mathrm{Al}_{2} \mathrm{O}_{3}$ & $\mathrm{MgO}$ & $\mathrm{CaO}$ & $\mathrm{S}^{2}$ & $\mathrm{WO}_{3}$ & $\mathrm{Others}$ \\
\hline Content (\%) & 9.45 & 48.22 & 5.01 & 2.96 & 12.68 & 2.75 & 0.055 & 18.875 \\
\hline
\end{tabular}

characteristics with the consolidation pressure and discussed the related mechanisms based on the microstructure parameters and soil compressibility obtained from the scanning electron microscope (SEM) images [4]. Liu et al. adopted a manual threshold method to obtain the basic parameters of pores such as porosity, fractal dimension, and nonuniform coefficient and analyzed the relationship between the microstructure and mechanics of rocks [5, 6]. Aldhafeeri, University of Ottawa, Canada, revealed the effect of oxygen consumption and temperature on the consolidation of CPB [7]. Aldhafeeri and Fall performed an oxygen-consuming experiment for sulfur-containing cemented tailings backfill and revealed its microscopic structure [8]. Wilson et al. of Massachusetts Institute of Technology analyzed a microstructure-tomacroscopic multiscale industrial clinker and the mechanical properties of Portland cement [9].

In contrast to the above, deep learning has been applied in image recognition, nonlinear processing, speech, and a few other fields [10-12]. Deep learning has a deep nonlinear network structure to achieve complex function approximation. Increasing the number of levels allows a more in-depth representation and more powerful function simulation. As the number of layers in the network increases, each level becomes deeper than the abstract level of the previous level $[13,14]$. Deep convolutional neural networks have led to a breakthrough in image processing, video, speech, and audio. They exhibit a good performance in solving the traditional nonlinear problems.

In the current work, the SEM image of cemented backfill captured at the microscopic scale is regarded as the input sample in the deep convolution neural network. The corresponding mechanical response is considered as the output. An end-to-end nonlinear prediction network model is established from the SEM images, which predicts the mechanical strength of the cemented backfill. The remainder of the paper is organized as follows. In Section 2, the experimental conditions pertaining to the materials and methods are described. In Section 3, basic principle of a convolutional neural network is explained. In Section 4, the constructed deep learning network and a specific experiment and analysis are presented. In Section 5, a summary is provided.

\section{Materials and Test Methods}

2.1. Material Components. Tailings were used in the basic performance test, and the measurement results of the main physical properties are listed in Table 1. The test samples of the main material are the full tailings of the Xiang furnace Shan tungsten mine, the strength grade of \#325-type Portland composite cement (PCC) and the urban tap water. Table 1 shows the 5 parameters, such as specific gravity, loose bulk density, dense bulk density, porosity, and natural rest angle. The grain composition and grain size distribution curve are presented in Table 2 and Figure 1, respectively. The gel material is ordinary Portland cement (OPC), and the test water is city tap water.

As can be seen from Figure 1, D10, D50, and D90 are the grain sizes of the tailings with the values of $3.63 \mu \mathrm{m}$, $52.667 \mu \mathrm{m}$, and $132.095 \mu \mathrm{m}$, respectively. The uniformity coefficient of the tail sand composition and the curvature coefficient are 16.52 and 0.65 , respectively. If the uniformity coefficient is extremely large, the natural grading of the tailings belongs to the category of discontinuous grading. If the coarse grain content of the test tailings is low, then it implies absence of coarse grains. The grains size distribution curve of the tail sand is shown in Figure 1. The main chemical composition and mass fraction of tailings were obtained by X-ray fluorescence spectrum analysis of tailings. The specific content is shown in Table 2.

The other quantitative analysis method is the XRD method, which predicts the chemical composition of the sample according to the ratio of the chemical elements. The XRD (D- MAX2200VPC, RIGAKU) analyses phase compositions, which uses a copper target, the tube pressure/pipe flow is $30 \mathrm{kV} \times(30 \mathrm{~mA})^{-1}$, the step width (relative to $2 \theta$ ) is 0.02 , and the scanning range is 3 to $80^{\circ}$. The analysis results of cemented backfill samples are shown in Figure 2. The background of combined tailings and cement production is compared with standard PDF card. The results of XRD analysis are as follows: the main products of cemented paste backfill samples are C-S-H, calcium hydroxide, and calcium silicate hydrate.

\subsection{Experimental Procedure}

2.2.1. Sample Preparation. The experiment requires the preparation of four identical samples. First, the quality of OPC required is calculated based on the lime-sand ratio, and then, it is weighed. According to the ratios given in Table 3, the appropriate amounts of the tailings and cement are weighed and mixed, and city tap water is added to prepare the sample (slurry mass fraction 76\%). A small mixer is used for mixing for more than $5 \mathrm{~min}$ until the cemented backfill is stirred uniformly. The interior of a cylindrical cast iron mold 


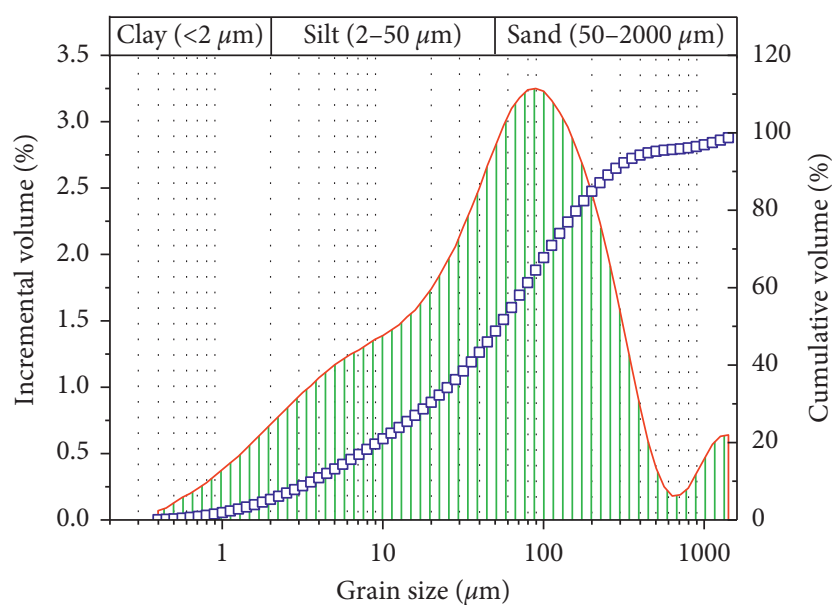

- - - Cumulative volume

पा Incremental volume

Figure 1: Grain size distribution curve of the tailings.

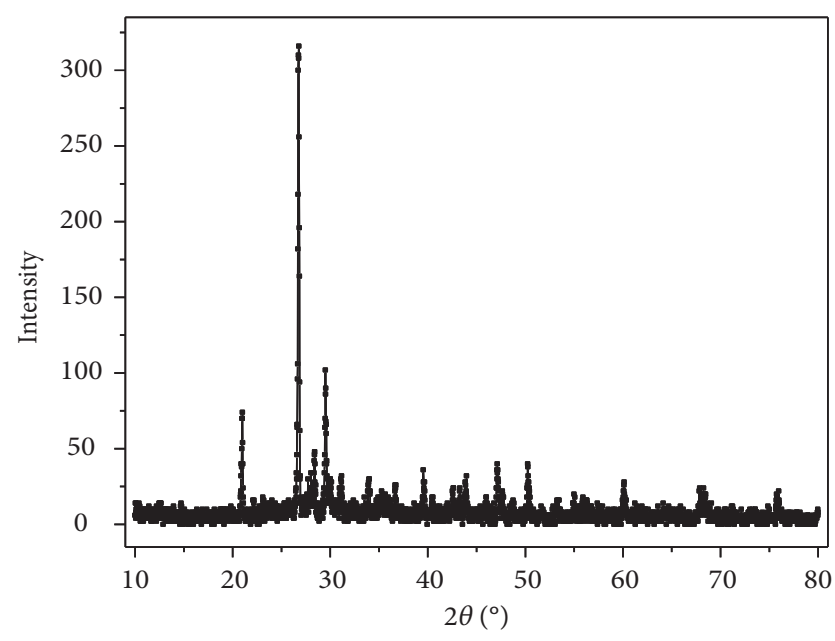

FIGURE 2: XRD pattern of CPB sample.

is coated with a layer of Vaseline. Its diameter and height are $50 \mathrm{~mm}$ and $100 \mathrm{~mm}$, respectively. The cemented backfill is divided into three layers in the mold, and each layer is then compacted by vibration. The $\mathrm{CPB}$ is then not moved for $24 \mathrm{~h}$ after being loaded. The surface of the test sample is then smoothed by scraping, and the mold is removed. The test samples are appropriately labeled and placed in a constanttemperature and constant-humidity curing box at a temperature of $(20 \pm 1)^{\circ} \mathrm{C}$ and humidity of $(95 \pm 1) \%$.

2.2.2. Test of Uniaxial Compressive Strength. Each test sample is removed, and its height and diameter are measured with a Vernier caliper 3, 7, 14, 28, and 56 days after curing. A computer-controlled $20 \mathrm{kN}$ pressure machine is employed to apply pressure at a constant rate of $1 \mathrm{~mm} / \mathrm{min}$ until the test sample fails, as shown in Figure 3. The data are then collated to compute the uniaxial compressive strengths of the test pieces, which are then averaged to obtain the result.
TABLE 3: Experimental design.

\begin{tabular}{lccc}
\hline Number & $\begin{array}{c}\text { Slurry concentration } \\
(\%)\end{array}$ & $\begin{array}{c}\text { Gelling } \\
\text { agent }\end{array}$ & $\begin{array}{c}\text { Cement-sand } \\
\text { ratio }\end{array}$ \\
\hline 1 & & OPC & \\
2 & 74 & OPC & $1: 6$ \\
3 & & OPC & \\
\hline
\end{tabular}

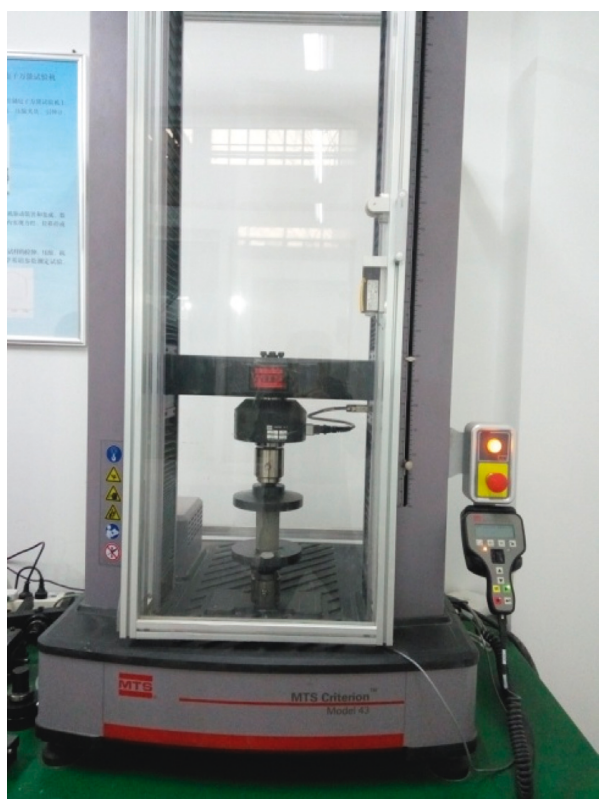

FIGURE 3: Test platform for measuring the uniaxial compressive strength of the CPB tailings.

2.3. Preparation of SEM Samples. In this research, SEM samples are fabricated. As a modern detection technology, a SEM has the characteristics of a high resolution, large magnification time, wide field-of-view, and strong effects of three-dimensional (3D) images. Consequently, the samples are required to be dried and gilded to obtain a true and clear observation.

The CPB is selected at different curing times for the preparation of the SEM test samples. First, the middle part of the cement backfill is located, and a double-sided blade wire saw coated with Vaseline is used to cut a $10 \mathrm{~mm} \times 10 \mathrm{~mm} \times 10 \mathrm{~mm}$ roughcast cube with a $1.5 \mathrm{~mm}$ border. A sharp backfill paste steel knife is then used to cut the blank and form a fresh cross section, baring a complete natural structural surface. This is then cut into a $5 \mathrm{~mm} \times 5 \mathrm{~mm} \times 5 \mathrm{~mm}$ block for observation under the electron microscope. For this, the fresh surface should be as flat as possible with all the disturbance particles removed by a rubber suction bulb.

\section{Principle of Deep Learning}

The concept of deep learning is to build a machine learning model with multiple hidden layers, learn more useful data from the massive training data, and improve the accuracy of the classification or prediction. Compared with traditional 
machine learning, its most prominent feature is a multilayer network structure, with an increased convolution and a down-sampling layer. The network is able to significantly reduce the number of parameters in the neural network training process because of weight-sharing characteristics. Furthermore, the down-sampling layer is invariant to displacement.

Generally, the convolution layer is connected to the input layer in a convolutional neural network (CNN). The input image is taken from the input layer of the network, and the middle layer is formed by alternately connecting the convolution layer and down-sampling layer. This implies that a down-sampling is performed once for each convolution operation, after which the convolution is continued. The complete connection structure is used after several convolutions and down-samplings, and finally, the output from the entire network is obtained. The output layer contains the target label information, such as people, cars, and animals. To reduce the computational complexity, CNNs share the neuron weights on the neuron plane, extract different features through different convolution kernels, effectively reduce the number of parameters in the training process of CNNs, and reduce the network complexity of parameter selection. In the convolutional layer, the feature map of the previous layer is convoluted with the convolution kernel, and the result forms the feature map of the next layer after the activation function. The feature map of the last layer is regarded as the input of the full connection layer, whereas the output of the full connection layer is considered as the final output. The main processes in the CNN training are as follows.

3.1. ConvolutionalLayer. The output of the $j$ th neuron in the convolution layer $\ell(1<\ell<N)$ is as follows:

$$
x_{j}^{\ell}=f\left(\sum_{i \in M_{j}} x_{i}^{\ell-1} * w_{i j}^{\ell}+b_{j}^{\ell}\right) \text {, }
$$

where $M_{j}$ represents a set of input feature maps (receptive field), $b$ denotes the offset corresponding to the output characteristic map, $f(\cdot)$ represents the activation function of the convolutional network, and $x_{j}^{\ell}$ represents the $j$ th neuron output at layer $\ell$. A convolution layer is usually composed of multiple feature maps, and the weights are shared among the feature maps to reduce the number of free parameters in the network.

3.2. SubsamplingLayer. A subsampling layer (the pool layer) usually follows the convolution layer, which can compress multiple pixel values into a single one. The average or maximum of the multiple pixels is usually chosen, and its function is to extract features to reduce the data size and spatial resolution of the network, leading to robustness against distortion and displacement. The calculation formula for the subsampled neurons is as follows:

$$
x_{j}^{\ell}=f\left[\beta_{j}^{\ell} \cdot \operatorname{pooling}\left(x_{j}^{\ell-1}\right)\right] \text {, }
$$

where pooling $(\cdot)$ represents a pooling function and $\beta$ is the weight coefficient.

3.3. Output Layer. The CNN training process can be divided into two stages, forward and backward propagation. In the forward propagation phase, information from the input layer is spread forward layer-by-layer. In this process, the calculation performed by the network is as follows:

$$
y_{i}=f_{i}\left(\cdots\left(f_{2}\left(f_{1}\left(X w_{1}+b_{1}\right) w_{2}+b_{2}\right) \cdots\right) w_{i}+b_{i}\right),
$$

where $y_{i}$ is the output of the $i$ th layer of the convolutional network, $f(\cdot)$ represents the activation function of the convolutional network, and $w_{i}$ is the weight of the $i$ th convolution kernel.

3.4. Backward Propagation Stage. In the backward propagation phase, the difference between the actual output and label information is calculated, and then the weight of each layer in the network is reversely propagated according to the strategy of minimizing error. The cost function, $E$, is given by

$$
\min E^{N}=\frac{1}{2} \sum_{n=1}^{N} \sum_{k=1}^{c}\left(t_{k}^{n}-y_{k}^{n}\right)^{2},
$$

where $E$ is the cost function, $c$ is the number of categories, $N$ is the number of samples, $t_{k}^{n}$ represents the $n$th sample corresponding to the target value of the $k$ th tag, and $y_{k}^{n}$ indicates the $n$th sample corresponding to the network of the $k$ th output.

Because the convolution layer is followed by a downsampling layer, the sensitivity of the down-sampled layer needs to be upsampled for ensuring that the size of the feature map is consistent. Then, the sensitivity is multiplied by the partial derivative of the feature map, and as in the down-sampling layer, the weight of the feature map is shared with a common weight, $\beta$ :

$$
\delta_{j}^{\ell}=\beta_{j}^{\ell+1}\left(f^{\prime}\left(u_{j}^{\ell}\right) \cdot \operatorname{up}\left(\delta_{j}^{\ell+1}\right)\right), \quad u_{j}^{\ell}=w_{j}^{\ell} x_{j}^{\ell}+b_{j}^{\ell},
$$

where up (.) is the upsampled operation and $\delta_{j}^{\ell}$ represents the sensitivity of the $j$ th neuron in the $\ell$ layer. The formula for calculating $\delta_{j}^{\ell}$ is as follows:

$$
\delta^{\ell}=\frac{\partial E}{\partial u} \frac{\partial u}{\partial b}=\left(W^{\ell+1}\right)^{T} \delta^{\ell+1} \cdot f^{\prime}\left(u^{\ell}\right) .
$$

3.5. Weight and Offset of Updating Process. The derivative of the error in each weight of the $l$ th layer is the cross product of the input of the current layer and sensitivity. The neuron weight update formula for this layer is obtained by multiplying the resulting partial derivative by a learning rate of $\eta$ :

$$
\begin{aligned}
\frac{\partial E}{\partial W^{\ell}} & =x^{\ell-1}\left(\delta^{\ell}\right)^{\mathrm{T}}, \\
\Delta W^{\ell} & =-\eta \frac{\partial E}{\partial W^{\ell}}, \\
\frac{\partial E}{\partial b} & =\sum\left(\delta^{\ell}\right) .
\end{aligned}
$$




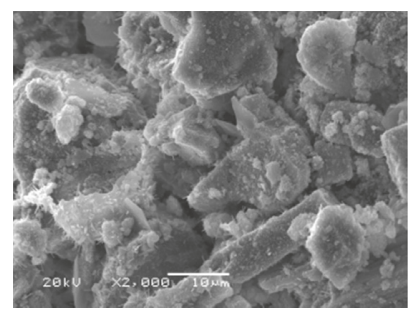

(a)

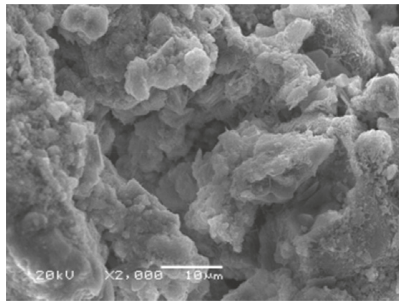

(b)

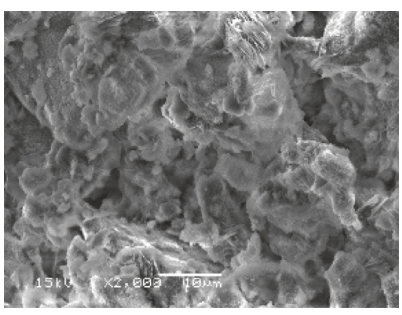

(c)

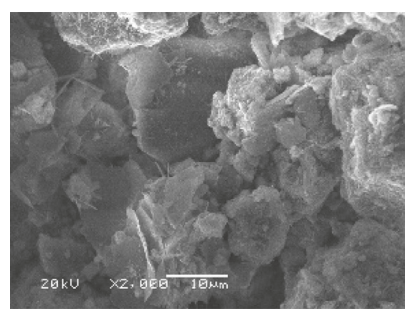

(d)

Figure 4: Microscopic scale SEM image sample set of the CPB.

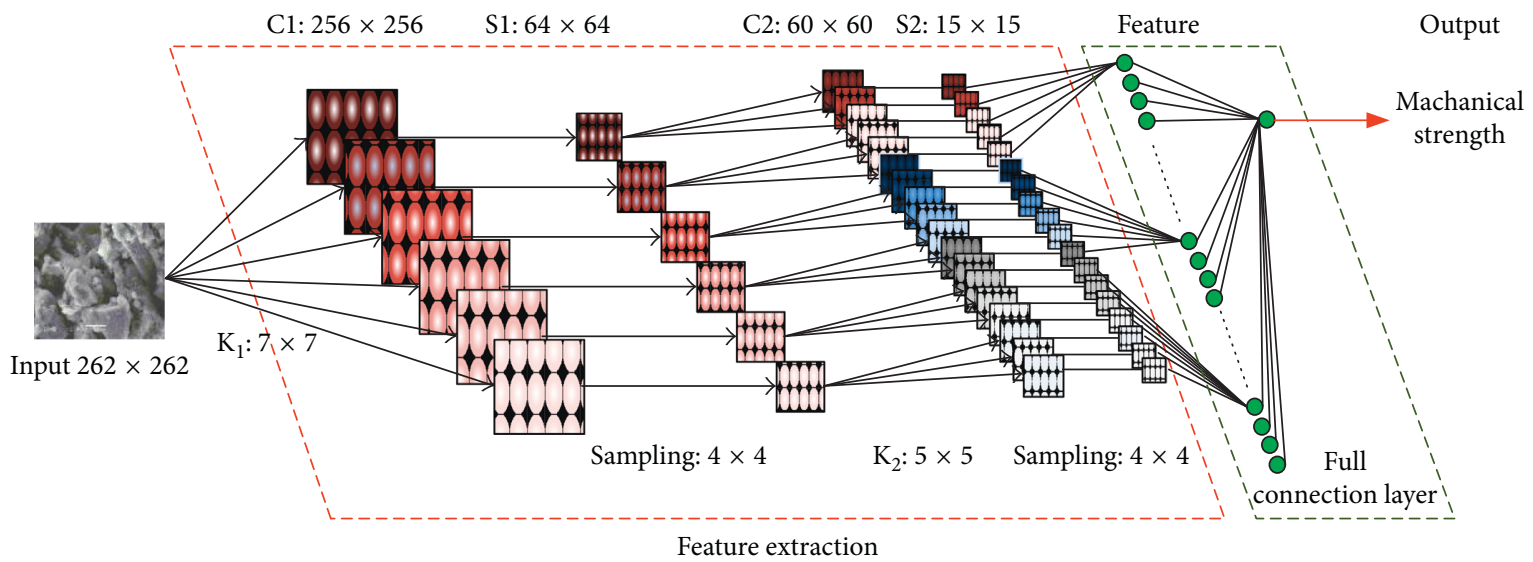

Figure 5: CNN structure diagram.

\section{Experiment and Result}

In the experiment, the SEM images of $3 \mathrm{~d}, 7 \mathrm{~d}, 14 \mathrm{~d}, 28 \mathrm{~d}$, and $56 \mathrm{~d}$ are used, and a seven-layer convolutional neural network structure is designed. The SEM image normalized to the size of 262 pixel $\times 262$ pixel is regarded as the input layer of the network. The output layer has a single node, which is the mechanical response strength. Figure 4 shows a sample set.

The CNN structure is also depicted in Figure 5. Here C1 and $\mathrm{C} 2$ are convolutional layers, $\mathrm{S} 1$ and $\mathrm{S} 2$ are the downsampling layers, and $\mathrm{K} 1$ and $\mathrm{K} 2$ are the convolution kernels. The network is a seven-layer CNN. The input image size is $262 \times 262$. Various convolution kernels represent different types of features. The input layer image (size: $262 \times 262$ ) is convolved with convolution kernel K1 (size: $7 \times 7$ ) to obtain C1 (size: $256 \times 256$ ) layer. Then convolution layer $\mathrm{C} 1$ is employed for the down-sampling operation to obtain downsampling layer S1 (size: $64 \times 64$ ). Similarly, the feature image is pooled and down-sampled to obtain the C2 and S2 layers. The objective is to further extract the features while reducing the computational complexity of the underlying convolution and then use the small convolution kernel and sampling layer convolution operation to derive small features. Further down-sampling is conducted to obtain all the features, and finally, full connectivity of these features uses simple neural networks to predict the mechanical strength to establish an end-to-end nonlinear relationship between the SEM image and mechanical strength.
TABLE 4: Comparison of the mechanical strength tested values and predicted values.

\begin{tabular}{lcc}
\hline Number & $\begin{array}{c}\text { Tested mechanical } \\
\text { strength } f_{v}(\mathrm{MPa})\end{array}$ & $\begin{array}{c}\text { Predicted strength } \\
p_{v}(\mathrm{MPa})\end{array}$ \\
\hline 1 & 0.361 & 0.321 \\
2 & 0.782 & 0.841 \\
3 & 0.898 & 0.945 \\
4 & 0.775 & 0.713 \\
5 & 0.732 & 0.701 \\
\hline
\end{tabular}

Next, the trained network is tested. During the training phase, 50 SEM images of each of $3 \mathrm{~d}, 7 \mathrm{~d}, 14 \mathrm{~d}$, $28 \mathrm{~d}$, and $56 \mathrm{~d}$ are taken, totaling 250 images. The sizes of all the images are normalized to 262 pixel $\times 262$ pixel and regarded as a training input sample set. The training output sample set is the corresponding mechanical response strength. During the training process, 4000 steps are iterated until the error of the training function is stabilized, indicating the completion of the training. During the test phase, 10 SEM images for each of $3 \mathrm{~d}, 7 \mathrm{~d}$, $14 \mathrm{~d}, 28 \mathrm{~d}$, and $56 \mathrm{~d}$ were taken as test samples. Table 4 lists tested mechanical strength $f_{v}$ and predicted strength $p_{v}$ of the five sets of the test samples.

The error function is defined as follows:

$$
\text { error }=\left|f_{v}-p_{v}\right| \text {. }
$$

The average error precision is defined as 
TABLE 5: Statistical indices of the error in the mechanical strength (50 sets of error data).

\begin{tabular}{lccc}
\hline $\begin{array}{l}\text { Average value of } \\
\text { error }(\mathrm{MPa})\end{array}$ & $\begin{array}{c}\text { Minimum value of } \\
\text { error }(\mathrm{MPa})\end{array}$ & $\begin{array}{c}\text { Maximum value of } \\
\text { error }(\mathrm{MPa})\end{array}$ & $\begin{array}{c}\text { Standard deviation value of } \\
\text { error }(\mathrm{MPa})\end{array}$ \\
\hline 0.0986 & 0.031 & 0.181 & 0.0756 \\
\hline
\end{tabular}

$$
a=\frac{\sum_{i=1}^{N}\left|f_{v i}-p_{v i}\right|}{\sum_{i=1}^{N} f_{v i}}
$$

where $f_{v i}$ is the $i$ th set of the actual measured values and $p_{v i}$ is the $i$ th set of the predicted values.

The analysis of the 50 sets of test sample data yielded error statistical indices, listed in Table 5, such as the average value, minimum and maximum, standard deviation, and accuracy. The average value of error is $0.8 \mathrm{MPa}$, minimum value is 0.2 , maximum value is 1.8 , and accuracy is $8.26 \%$.

\section{Conclusion and Discussion}

Cement, tailings, water, and other materials were fully mixed, and the fixed slurry concentration and cement-sand ratio of CPB were obtained. An SEM specimen using CPB was fabricated, and the corresponding SEM image was captured. The mechanical strength of the cemented filling was predicted by the method of deep learning. A seven-layer convolution neural network was built, where the input layer was an SEM image, whereas the output node was the mechanical strength of the cemented backfill. A nonlinear predictive model of the cemented backfill with SEM was established to derive the mechanical strength. The difference between the test and predicted strengths of the cemented backfill was calculated, and the mean and square difference of the statistical parameters of the error were analyzed, yielding an average error with the accuracy of $8.28 \%$.

The method presented in this paper provides a new technique and concept for predicting the mechanical strength of cemented backfill. Because the SEM images of cemented backfill were in the microscopic scale, further work will be devoted for extracting the characteristic images in the microscopic scale of cemented filling to be used as input in the deep learning network and to improve the prediction precision of the mechanical strength of cemented backfill.

\section{Data Availability}

The data used to support the findings of this study are included within the article.

\section{Conflicts of Interest}

The authors declare that there are no conflicts of interest regarding the publication of this paper.

\section{Acknowledgments}

This research was supported by the National Natural Science Foundation of China (nos. 51704229, 51504182, 51674188, 51404191, and 51405381), the Natural Science Basic Research Plan of Shaanxi Province of China (no. 2015JQ5187), the
Scientific Research Program funded by the Shaanxi Provincial Education Department (nos. 15JK1466 and 15JK1472), the project funded by China Postdoctoral Science Foundation (no. 2015M582685), and the Industrial Science and technology research foundation of Shaanxi province (no. 2016GY040).

\section{References}

[1] T. Luo, The Study of the Damage and Infrasonic Characteristics in Uniaxial Compressive of the Cemented Tailings Backfill, Jiangxi University of Science and Technology, Ganzhou City, Jiangxi Province, People's Republic of China, 2016.

[2] Z. Qinli, X. Li, and W. Yang, "Optimization of filling slurry ratio in a mine based on back-propagation neural network," Journal of Central South University (Science and Technology), vol. 44, no. 7, pp. 2867-2874, 2013.

[3] W. Xu, X. Tian, Q. Yu, D. Peng, and Y. Tianjun, "Experiment of the resistivity characteristic of cemented backfill mass during the whole consolidation process," Journal of China University of Mining and Technology, vol. 46, no. 2, pp. 265-272, 2017.

[4] J. Zhou, Y. Deng, Y. Cao, and J. Yan, "Experimental study of microstructure of Hangzhou saturated soft soil during consolidation process," Journal of Central South University (Science and Technology), vol. 45, no. 6, pp. 1998-2005, 2014.

[5] C. Liu, B. Shi, J. Zhou, and C. Tang, "Quantification and characterization of microporosity by image processing, geometric measurement and statistical methods: application on SEM images of clay materials," Applied Clay Science, vol. 54, no. 1, pp. 97-106, 2011.

[6] C. Liu, C.-S. Tang, B. Shi, and W.-B. Suo, "Automatic quantification of crack patterns by image processing," Computer and Geosciences, vol. 57, no. 2, pp. 77-80, 2013.

[7] Z. Aldhafeeri, M. Fall, M. Pokharel, and Z. Pouramini, "Temperature dependence of the reactivity of cemented paste backfill," Applied Geochemistry, vol. 72, pp. 10-19, 2016.

[8] Z. Aldhafeeri and M. Fall, "Sulphate induced changes in the reactivity of cemented tailings backfill," International Journal of Mineral Processing, vol. 166, no. 10, pp. 13-23, 2017.

[9] W. Wilson, Grinding of Cement Clinkers: Linking Multi-Scale Fracture Properties to System Chemistry, Mineralogy and Microstructure, Massachusetts Institute of Technology, Cambridge, MA, USA, 2013.

[10] M. M. Al Rahhal, Y. Bazi, H. AlHichri, and R. R. Yager, "Deep learning approach for active classification of electrocardiogram signals," Information Sciences, vol. 345, pp. 340-354, 2016.

[11] D. M. Tissera and D. M. Mark, “Deep extreme learning machines: supervised autoencoding architecture for classification," Neurocomputing, vol. 174, no. 2, pp. 42-49, 2016.

[12] S. Roy, N. Das, M. Kundu, and M. Nasipuri, "Handwritten isolated Bangla compound character recognition: a new 
benchmark using a novel deep learning approach," Pattern Recognition Letters, vol. 90, pp. 15-21, 2017.

[13] H. Li, J. Chen, H. Lu, and Z. Chi, "CNN for saliency detection with low-level feature integration," Neurocomputing, vol. 226, pp. 212-220, 2017.

[14] E. P. Ijjina and K. M. Chalavadi, "Human action recognition using genetic algorithms and convolutional neural networks," Pattern Recognition, vol. 59, pp. 199-212, 2016. 


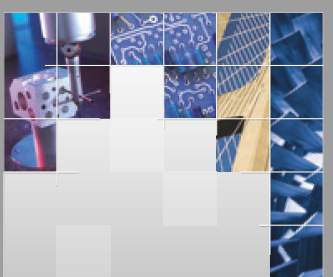

\section{Enfincering}
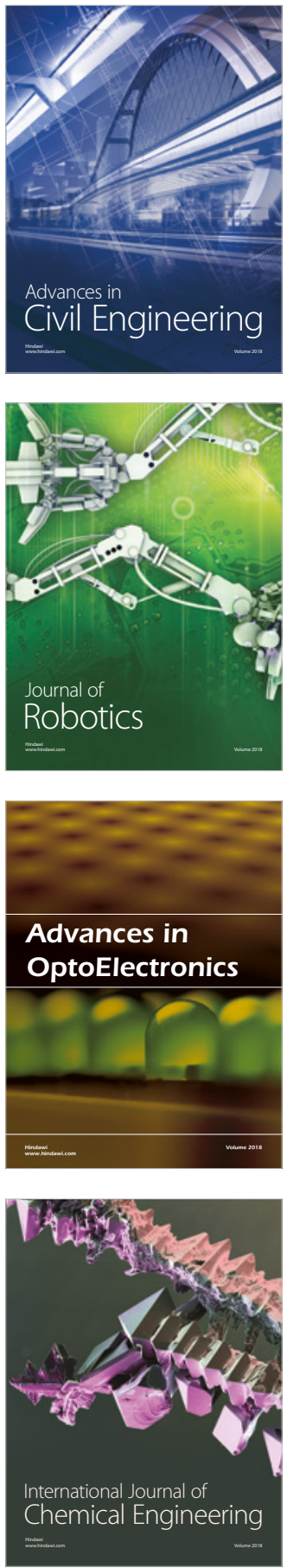

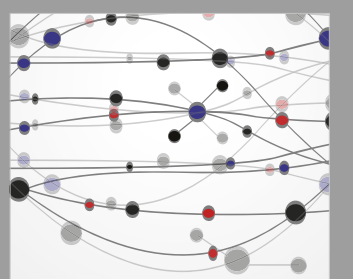

\section{Rotating \\ Machinery}

The Scientific World Journal

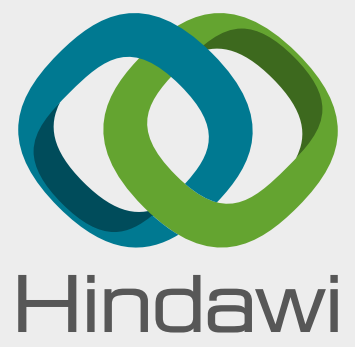

Submit your manuscripts at

www.hindawi.com
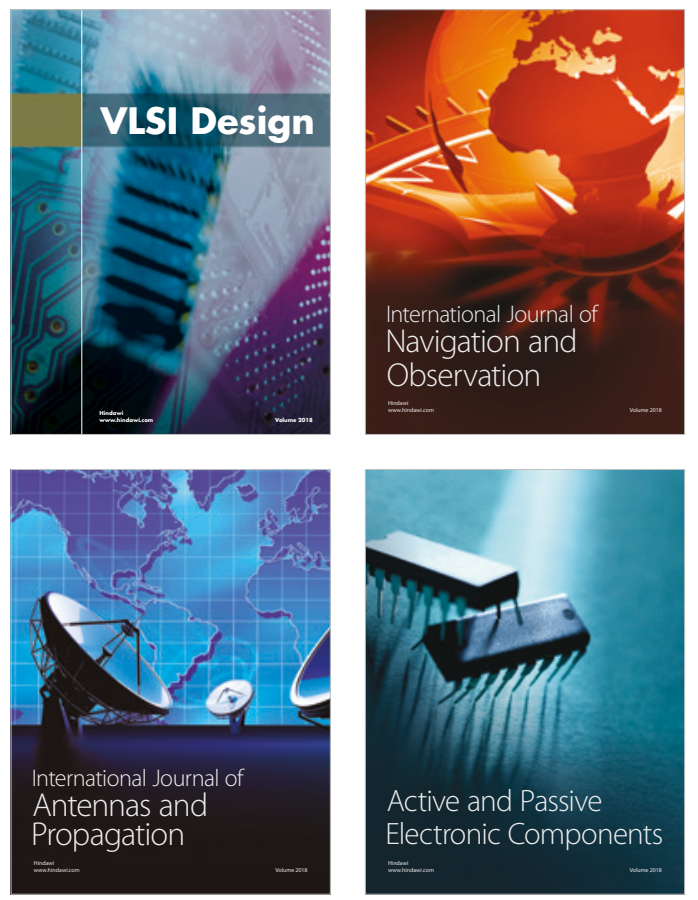
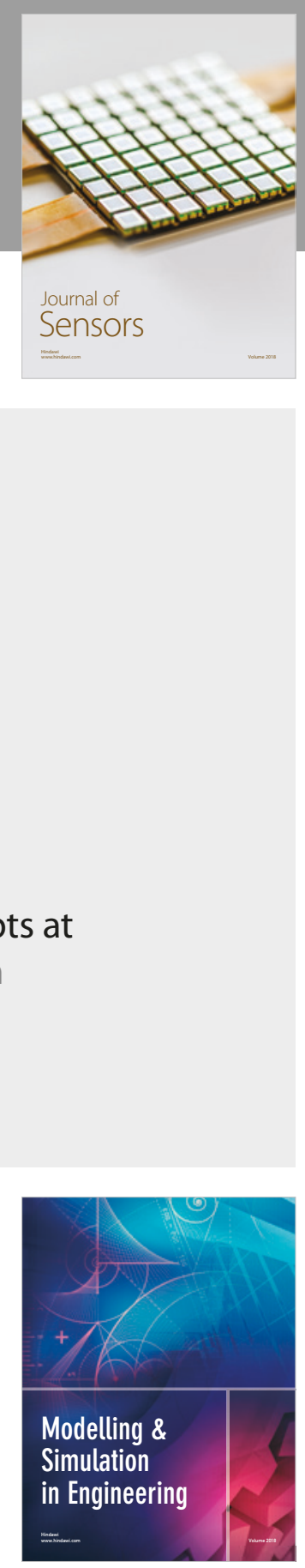

\section{Advances \\ Multimedia}
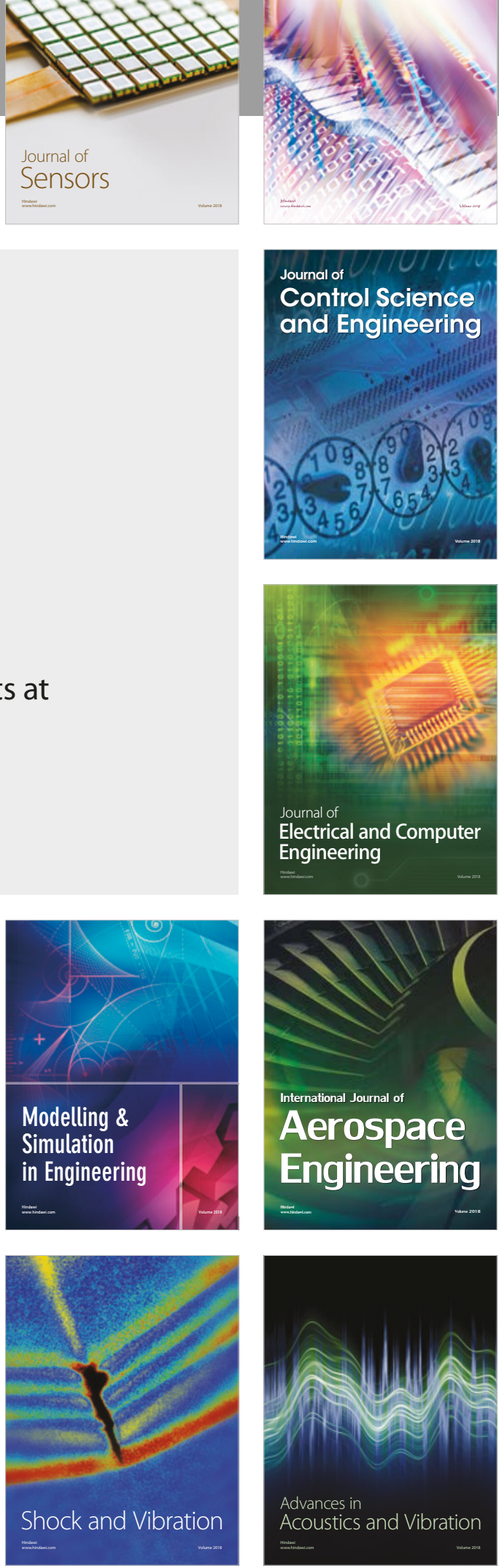